\title{
COMPRA TECNOLÓGICA Y FLUJOS EN ANDALUCÍA
}

\author{
Rosa María Jordá Borrell \\ Francisca Ruiz Rodríguez \\ Departamento de Geografía Física y Análisis Geográfico Regional. Universidad de Sevilla \\ borrell@us.es, fruiz@us.es
}

\section{RESUMEN}

Con los datos que proporciona una encuesta se estudia la compra de tecnología de las empresas innovadoras andaluzas en el exterior de la región, especialmente a los países desarrollados. Los objetivos se centran en el análisis del volumen de la compra tecnológica, el proceso de innovación-transferencia y los factores sectoriales y territoriales que inciden. Se hace un análisis descriptivo, factorial y cluster a una muestra de 193 empresas. A través del análisis cluster se distinguen seis grupos de empresas. En Andalucía, el proceso de innovación empresarial, la transferencia tecnológica y el éxito en el mercado está asociado a la concentración geográfica de empresas, especialmente en las áreas metropolitanas.

Palabras claves: compra de tecnología, proceso de innovación, territorio.

\begin{abstract}
Based on data provided by a survey we studied the acquisition of innovative technology performed by Andalusian corporations from other spanish regions and overseas, paying special attention to those obtained from developed countries. Our goals focus on analyzing the intensity of technology purchasing, the process innovation-transfer and the factors which influence sectorial and territorial. Thus, we carry out a descriptive analysis, as well as factor and cluster analysis to a sample of 193 companies. Thruough the cluster analysis there are six groups of companies. In Andalusia, the process of business innovation, technology transfer and market success is related to the geographic concentration of firms, namely in metropolitan areas.
\end{abstract}

Key words: Technological purchase, process of innovation, territory.

Fecha de recepción: febrero 2011.

Fecha de aceptación: julio 2012. 


\section{INTRODUCCIÓN}

En las últimas décadas, la emergencia de la sociedad del conocimiento caracterizada por el uso de intangibles y de tecnologías avanzadas (NTICs), de la globalización de los procesos productivos fundamentados en la introducción de avances tecnológicos, han convertido a la capacidad innovadora y a la compra de tecnología de las empresas en uno de los principales factores de crecimiento económico a diferentes escalas territoriales (Lichtenberg/Siegel, 1991; Fageberg, 1994; Freeman, 1994). Paralelamente la globalización y la liberalización económica han acelerado la internacionalización de las empresas, incluso en regiones europeas de convergencia, sobre todo en aquellas que han conocido un mayor dinamismo a través de la innovación. De forma que estos procesos se han convertido en los factores explicativos más importantes del cambio de sus estructuras productivas (regionales y locales).

La necesidad de transformar a los territorios innovadores en sistemas abiertos y adaptativos va ligado al desarrollo de los procesos de internacionalización de las empresas a distintos niveles (exportación, inversión directa extranjera, uso de licencias, franquicias, etc.) y a la capacidad de las regiones de acumular numerosos contratos de compraventa ${ }^{1}$ de tecnología con entidades de otros países (Jordá y Ruiz, 2009) al objeto de incrementar el nivel de competitividad y de desarrollo. Todo ello es debido a que el proceso de innovación de las empresas se basa en la adquisición de tecnología externa y en el desarrollo interno de I+D para generar tecnología propia. De hecho ambas fases tecnológicas se complementan. La empresa compradora ha de asimilar la tecnología adquirida para poder aplicarla, adaptarla a la propia y convertirla en un nuevo producto, produciéndose transferencia tecnológica. Pero, se necesita que la tecnología adquirida sea compatible con sus bases previas de conocimiento (Fernández, 2005).

En realidad, los contratos de compraventa entre los distintos agentes económicos de uno o varios territorios requieren el desarrollo de capacidades (Cohen y Levinthal, 1990, Arvanitis, 2003) por parte de las empresas. Una de las más importantes es la de absorción, al objeto de identificar, asimilar y explotar el conocimiento del exterior con la meta de conseguir y sostener una ventaja competitiva (Lankhuizen, 2006; Kim, 2000). Así pues, el proceso de creación de capacidades permite definir a las empresas como «instituciones, almacenes y fuentes de conocimiento» y por extensión, a los territorios cómo «inteligentes», es decir, sistemas socio-técnicos, abiertos, capaces de aprender y de transformarse permanentemente para adaptarse a los cambios externos e internos. En definitiva, los contratos de compraventa de tecnología se convierten en un problema de adquisición, de aprendizaje y de apropiación (Arvanitis, 2003), definición que se podrá aplicar al territorio y a la firma.

1 El contrato de ventas o compras de licencias de tecnología, es un acto por el cual una persona, natural o jurídica, transfiere a otra persona (comprador), natural o jurídica, un conocimiento o conjunto de conocimientos útiles para el logro de fines. En este contrato el licenciante es el propietario de la licencia y el licenciatario es el titular de la licencia al cual se le transfieren los elementos del conocimiento técnico que son necesarios para la concepción, diseño, construcción y operación de unidades que producen bienes y servicios. Es decir, las actividades inherentes al derecho de patente como podrían ser el uso o la explotación de dicha tecnología. De esta forma, el acuerdo de licencia es un arrendamiento de propiedad intangible. Es un acuerdo mediante el cual en lugar de dar el derecho de uso de bienes materiales, se confiere el uso de derechos de propiedad industrial e intelectual. Este contrato puede ser exclusivo o no. Con este contrato, el licenciante no pierde la propiedad. 


\section{OBJETIVOS, APARATO TEÓRICO, HIPÓTESIS Y METODOLOGÍA}

Dado que las empresas innovadoras son las que más tecnología adquieren en el exterior de Andalucía, y las que más innovaciones crean, favoreciendo así el crecimiento/desarrollo de las ciudades/núcleos, los objetivos principales de este trabajo son: 1.- Medir la magnitud de los flujos de compra de tecnología de las empresas innovadoras con el exterior de la región, especialmente con los países desarrollados, pues allí se supone que se adquiere la más compleja y avanzada. 2. Comprobar si existe correlación entre la intensidad de la compra de tecnología y el proceso de innovación-transferencia tecnológica en el territorio andaluz. 3.Descubrir los factores mediante análisis factorial que influyen en la compra de tecnología a fin de conocer las variables clave de este proceso; 4.- estudiar donde se concentran las firmas que compran tecnología (áreas metropolitanas, ciudades medias o en los núcleos rurales); 5. Distinguir grupos de empresas a través de análisis cluster según su comportamiento ante la adquisición externa de tecnología y su capacidad de transferir dicha tecnología al territorio.

Para alcanzar estos objetivos se debe tener en cuenta que la introducción de una nueva tecnología mediante compra representa la adquisición de un activo, la aplicación de una nueva tecnología en la firma compradora, el desarrollo de I+D y de tecnología propia en ésta última, la probabilidad de generar nuevas relaciones de tipo técnico con el entorno territorial, de adquirir nuevas competencias, de desarrollar nuevos productos; en conclusión, de ampliar la red a través de la cual se conecta la firma compradora con su entorno territorial. De ahí que es conveniente que la empresa que adquiere tecnología disponga de habilidades como knowhow, lenguaje compartido (comprador-vendedor) y conocimiento técnico que le permita absorber el conocimiento transferido (Fernández, 2005).

Lo importante en un contrato de compraventa de tecnología es que exista entre las distintas entidades proximidad cultural, tecnológica y organizativa, mientras que la cercanía física es menos relevante. Las tecnologías se intercambian por medio de la transferencia simultánea de un bien tangible o intangible y de algunos servicios asociados (formación, asistencia en el diseño, etc.). Ello se explica porque el comprador debe aportar activos propios al proceso para que la tecnología se adapte mejor a sus necesidades y a la tecnología propia (I+D y otras actividades innovadoras). De forma que la tecnología adquirida y la propia se deben complementar y/o ser compatibles (Dixón, 2000).

Por otra parte, el tamaño de las ciudades (según población) donde se localizan las empresas constituye un factor clave en la compra de tecnología y en la generación de transferencia tecnológica debido a la necesidad de las firmas de economías externas, entendiendo que a mayor tamaño más volumen de éstas últimas. En este trabajo se parte de la definición del Instituto Nacional de Estadística de España (INE) sobre núcleo urbano (aquel que tiene más de 10.000 habitantes) y considera municipio rural cuando no llegan a dicho límite. Determinar los intervalos de población de una ciudad media es difícil, incluso para el caso español (Méndez, 2007; Caravaca, 2005) dado que no existe un consenso por parte de los autores de cuales deben de ser estos, aunque se suele establecer como límites el intérvalo de los 10.000 a los 250.000 habitantes. Concretar los umbrales de población de una aglomeración resulta complejo, el Banco Mundial define una gran ciudad a partir de un millón de habitantes, la Unión Europea desde los 500.000 (Precedo, 1996) y en España se da diversidad de opiniones aceptándose, siempre, un cierto grado de arbitrariedad en la definición (Ganau y Vilagrasa, 2003). 
Andalucía en sus $87.598 \mathrm{Km}^{2}$ de superficie (17,4\% de España y el 3,7\% de la Unión Europea) cuenta con 770 municipios de los que unos 150 tienen una población superior a 10.000 habitantes y centralizan el $80 \%$ de la población. Por lo tanto, la región posee un modelo territorial concentrado con 2.707 núcleos de población que albergan un total de 8.415.490 habitantes (Avance Padrón 2011, INE), representando el 17,8\% de la población española. En consecuencia, y de acuerdo con las características del sistema urbano andaluz, se catalogan los núcleos de menos de 10.000 habitantes como rurales, entre 10.000 y 100.000 ciudades medias y más de 100.000 aglomeraciones urbanas o grandes ciudades según los umbrales establecidos por el gobierno autónomo regional (Consejería de Obras Públicas y Transporte, 1999) y el estudio de comarcalización llevado a cabo por Cano García (2002).

Además, cabe pensar que la gran parte de las empresas que han adquirido tecnología (extranjero, Andalucía o España) a pesar de que la mayoría están internacionalizadas, están integradas en el territorio andaluz (a través de relaciones con el tejido empresarial) vía mercado (proveedores y/o clientes o mediante transacciones tecnológicas) generando redes. En este sentido, Dematteis (1995) señala que la dinámica de los nodos de una red (ciudades, empresas...) depende de los enlaces funcionales con éstas últimas y de una componente endógena local y/o global. En realidad, las relaciones vienen definidas por el alcance del mercado de las empresas localizadas en esos nodos (local, regional, global), por cuestiones sectoriales (industria de alta tecnología, de baja tecnología...), por el tamaño de las economías externas y por el tipo de sinergias que mantienen con el resto del sistema productivo, científico y tecnológico, para transferir o comprar tecnología y conocimiento, según el grado de innovación alcanzado por las firmas (gestión de calidad, ingeniería, diseño, I+D).

Las características del sistema territorial de producción (STP) definen la transmisión interna de información y conocimientos, y por tanto, los procesos de aprendizaje. Maillat y Kebir (1998) estudian los STP a partir la combinación de dos criterios: a) las relaciones, o no, de interdependencia entre las empresas y su entorno local/regional; y b) el tipo de sinergias dominantes de las empresas (o grado de integración de su cadena de valor).

Así pues, las economías externas constituyen un activo intangible de ámbito local o comarcal que no pueden adquirirse en el mercado y del que se benefician las firmas localizadas en dicho territorio. Lo que se traduce en una mayor facilidad para llevar a cabo procesos de aprendizaje colectivo y difusión del conocimiento y de la tecnología a escala local y regional (Jordá y Ruiz, 2009). En realidad, la industria y los servicios se concentran en los territorios que ofrecen ventajas a las empresas. El tamaño del núcleo, la ubicación o proximidad a un área metropolitana, el ser sede político-administrativa, el volumen de economías externas ofrecidas por éstos y la existencia de universidad constituyen factores interrelacionados que mejoran extraordinariamente el desarrollo de la innovación, la transferencia tecnológica y facilitan la compra de tecnología en el exterior.

Por otra parte, la empresa innovadora internacionalizada que compra tecnología está asociada a la exportación directa e indirecta (proveedora de inputs a empresas foráneas) y a la importación de inputs especializados en el mercado internacional (Jordá y Ruiz, 2009). Lo que viene a demostrar: a) que los procesos innovadores de estas empresas no han conseguido todavía vincularse mucho a la capacidad de internacionalización con fórmulas de deslocalización de plantas, pero si mediante la subcontratación, compra de productos de alta tecnología y como proveedor de productos de media y media-alta tecnología. Las exigencias 
del cliente obliga a establecer relaciones tecnológicas y a aumentar las capacidades técnicas del proveedor a fin de desarrollar procesos y productos nuevos, requiriendo por tanto colaboración tecnológica (Jordá y Relaño, 2009) y; b) siguiendo las teoría de encadenamientos de Hirschman y la de la cadena de valor (Lucendo, 2009), los factores a nivel regional u otras escalas para la inserción o el desarrollo de proveedores tecnológicos locales dependen: 1. del tamaño y crecimiento de la demanda, su estructura y concentración geográfica; y 2. de factores internos de los proveedores (competitividad y estrategia de inserción). En cualquier caso, para realizar este estudio se partió de un conjunto de criterios, entendidos como principales formas genéricas de implantación internacional (de mayor a menor complejidad) siguiendo a Root (1994) y Pla y León (2004), y que se adaptó a las características de las firmas localizadas en Andalucía (Jordá y Ruiz, 2009):inversiones directas en el exterior (IED); acuerdos contractuales (licencias o franquicias internacionales) por el cual se transfiere un derecho de propiedad (patente, marca, know how, etc.); exportación directa; exportación indirecta (proveedores de multinacionales) e importación indirecta (subcontratación de segmentos del proceso productivo o la contratación de proveedores extranjeros).

De acuerdo con este contexto teórico y metodológico hemos partido de las siguientes hipótesis:

1. La adquisición de tecnología externa a Andalucía y la realización de I+D interna de la empresa compradora para generar tecnología propia constituyen dos etapas tecnológicas complementarias de la firma.

2. Los contratos de compraventa entre diferentes agentes en uno o varios territorios requieren el desarrollo de capacidades y una de las más importantes es la de absorción.

3. Las empresas innovadoras internacionalizadas son: a) las que más tecnología adquieren en el exterior de Andalucía, especialmente en los países desarrollados; y b) las que más innovaciones crean, favoreciendo así el crecimiento/desarrollo de las ciudades/ núcleos a partir de los procesos de transferencia tecnológica.

4. Las economías externas, representadas por las firmas de servicios avanzados, favorecen la concentración espacial de empresas, pero como su intensidad tecnológica en Andalucía es de tipo medio-bajo (INE, 2002), contribuyen poco a elevar el nivel tecnológico de la región en comparación con lo que sucede en los países desarrollados.

En esta investigación, se parte de una muestra de 263 empresas innovadoras que representa al total de firmas innovadoras andaluzas (el Directorio de Empresas Innovadoras Andaluzas, DEIA, contabiliza 1919 empresas $^{2}$, seleccionadas con la técnica de muestreo estratificado de forma que recoge la multiplicidad de características que aquellas presentan (tamaño, rama de actividad, capacidad tecnológica, localización territorial, etc.). Con los anteriores estratos se realizó el cálculo del tamaño de la muestra representativa. Mediante la norma de afijación óptima se tuvo en cuenta la población de cada estrato y la dispersión interna de las empresas que había en los mismos. Según este criterio, a algunas ramas de actividad no le correspondió ninguna empresa a encuestar lo que permitió identificar qué

2 Elaborado y actualizado anualmente por profesores (entre ellos los firmantes) del Grupo de Investigación Estudios Geográficos Andaluces 
sectores podían ser desestimados. A continuación se realizó un segundo cálculo del tamaño de la muestra escogiendo los estratos a partir del cruce de las variables sector y localización espacial, pero en este caso sólo considerando aquellos sectores significativos.

La información para la consecución de los objetivos se extrae de una encuesta-entrevista (de una hora y media de duración) que se pasó a las empresas innovadoras localizadas en Andalucía a lo largo del 2009-10, financiada por un proyecto de investigación del Ministerio de Educación y Ciencia (MEC). De éste se seleccionaron un conjunto de 88 variables (de un total de 650) que se relacionan con la compra de tecnología de la empresa (número y gasto de tecnología adquirida, localización y sector del proveedor, tiempo en adoptar las tecnologías adquiridas, en aplicarlas, desarrollarlas y el tiempo de adopción de éstas por sus competidores, tipos de acuerdos implícitos en los contratos de compra), características generales (facturación, localización, capital de su empresa, sectores según la clasificación de Pavitt y Soete-Miozzo ${ }^{3}$, mercado, etc.), capacidades o recursos principales de las empresas (importancia de los recursos humanos, organizacionales, tecnológicos y geográficos), organización general (tipo de organización, planificación estratégica, registro de conocimientos), organización del trabajo (papel de las rutinas, mecanismos de comunicación), relaciones con proveedores (tipos de intercambio -maquinaria, inmateriales, electrónico, etc.- según gastos) e innovación (gasto de I+D, diseño, ingeniería, otras actividades innovadoras, desarrollo de tecnologías, propias). Y por otro lado, se han identificado 193 empresas que compran tecnología de un total de 263 encuestadas, de las que 128 están internacionalizadas. Comprobándose con las técnicas de muestreo que se suelen emplear en estos casos, que la muestra es representativa de la población objeto de estudio.

Con la información suministrada se ha construido una base de datos al objeto de 1.gestionar la información con Access y Excel, para llevar a cabo análisis previos y efectuar un tratamiento estadístico descriptivo de la compra de tecnología por parte de las empresas innovadoras 2.- realizar un análisis factorial para identificar interdependencia o interrelaciones entre variables. Una vez que se conoció los principales flujos de compra de tecnología y los componentes que influyen en dicha compra a través del análisis factorial se obtuvieron grupos de empresas/establecimientos homogéneos por medio del análisis cluster, basándonos en las puntuaciones factoriales. Estos análisis permitieron estudiar y establecer una taxonomía de empresas compradoras de tecnología y explicar los flujos existentes en cada uno de los grupos.

\section{EL PESO DE LAS GRANDES CIUDADES EN LA COMPRA DE TECNOLOGÍA FORÁNEA}

Según el resultado de las encuestas, el $73 \%$ de las empresas innovadoras han comprado tecnología. Por término medio éstas la adquieren en Europa (52,7\% del gasto efectuado), el Resto del Mundo (26,5\%), Andalucía (11,9\%) y España $(8,9 \%)$. El análisis del comportamiento de las firmas innovadoras demuestra que su proceso de modernización y cam-

3 Pavitt (1984) clasifica a las empresas industriales según patrones de innovación en: Dominados por los proveedores (DP), Productores a gran escala (PGE), Proveedores Especializados (PE) y de Base Científica (BC); Soete-Miozzo (1989) adapta esta clasificación para el sector servicios, y distingue: Dominados por proveedores (DP), Servicios Intensivos en Producción, en Escala y Servicios de Redes (IPER) y Proveedores Especializados en Tecnología y Sectores de Base Científica (PETBC). 
bio tecnológico ha requerido adquirir tecnología externa, fundamentalmente en los países desarrollados: patentes (13\% del gasto), know-how (18\%), plantas llaves en mano (19,5\%), bienes de equipo (34\%) software $(6 \%)$, marcas $(9 \%)$, etc. También se ha confirmado a través de un índice de correlación $(0,981)$ que el proceso anterior va acompañado de un desarrollo interno de tecnología a través de I+D (65\% de las firmas) y de otras actividades innovadoras $(70 \%)$, debido a que ambas fases tecnológicas se complementan. Las encuestas indican igualmente que el proceso de vinculación de la tecnología adquirida a la propia, incluyendo la generación de innovaciones incrementales tiene una duración media de 15 meses, siendo este periodo más largo que el promedio español. Estas empresas se concentran especialmente en áreas metropolitanas y capitales de provincia $(70,98 \%)$ por la necesidad de economías externas, representadas en esta investigación por los servicios avanzados que tienen también una intensidad anual de I+D medio-baja $(547.676,6 €)$, inferior a la media nacional (791.754,5€).

De las firmas que desarrollan I+D, el $20,5 \%$ adquieren nuevos conocimientos y concentran sus esfuerzos en asimilarlos mediante la capacidad de absorción potencial (Zahra y George, 2002), lo que sólo les permite renovar sus stocks de conocimiento y conocer sus entornos territoriales; pues, el que una firma evalúe y adquiera conocimiento del exterior no garantiza la explotación de éste. Sin embargo, las empresas que se centran en la transformación y en la explotación $(79,5 \%)$ de la tecnología adquirida lo hacen con la capacidad de absorción realizada, aunque pueden no ser capaces de responder a los cambios del entorno territorial sino han generado habilidades para adquirir y asimilar conocimiento (Jiménez, Molina y García, 2009) y más cuando no cuentan con políticas eficientes para desarrollar un mercado tecnológico amplio.

Por lo tanto, la filtración de esas tecnologías en la empresa vía capacidad de absorción potencial y/o realizada favorece el aumento de la base de conocimiento empresarial y el nivel tecnológico regional. Pero, en el caso andaluz, el número de empresas compradoras de tecnología, el volumen y complejidad de ésta, así como el desarrollo de I+D interno no es suficiente para llevar a cabo avances tecnológicos al mismo nivel técnico que lo efectúan los países desarrollados. Según gastos realizados, la empresa innovadora andaluza (263 según muestra) se abastece tecnológicamente en un $41,2 \%$ en la región (se incluye la compraventa de productos innovadores, cooperación y la adquisición de tecnología). Sin embargo, cuando se analiza únicamente las firmas que han efectuado compra (193) de tecnología (patentes, modelos de utilidad, Know-how, bienes de equipo) este porcentaje desciende a $28,7 \%$.

Este hecho obedece a: 1) que el 66,7\% de las empresas están internacionalizadas, insertas en cadenas productivas globales, lo que explica en parte la adquisición de tecnología en el extranjero; 2) a la escasez de registro de patentes y otras figuras de protección en Andalucía, ya que según la muestra utilizada en este trabajo solamente el 30\% de las firmas han registrado tecnología propia; y 3) al reducido mercado de tecnología regional donde únicamente el $20 \%$ de las empresas venden tecnología y sólo un 30\% compran dentro de la región. De ahí se deduce que la intensidad de I+D de los servicios avanzados es medio-baja, aún existiendo un sistema $\mathrm{I}+\mathrm{D}+\mathrm{i}$, concentrado en las áreas metropolitanas y capitales de provincia (70,98\%) junto con una fuerte aglomeración de servicios avanzados $(71,10 \%)$ incluido las universidades, suponiendo la compra de tecnología externa en estas áreas el 80,0\% del gasto realizado en Andalucía. De la misma manera, en las ciudades medias donde se localiza el 


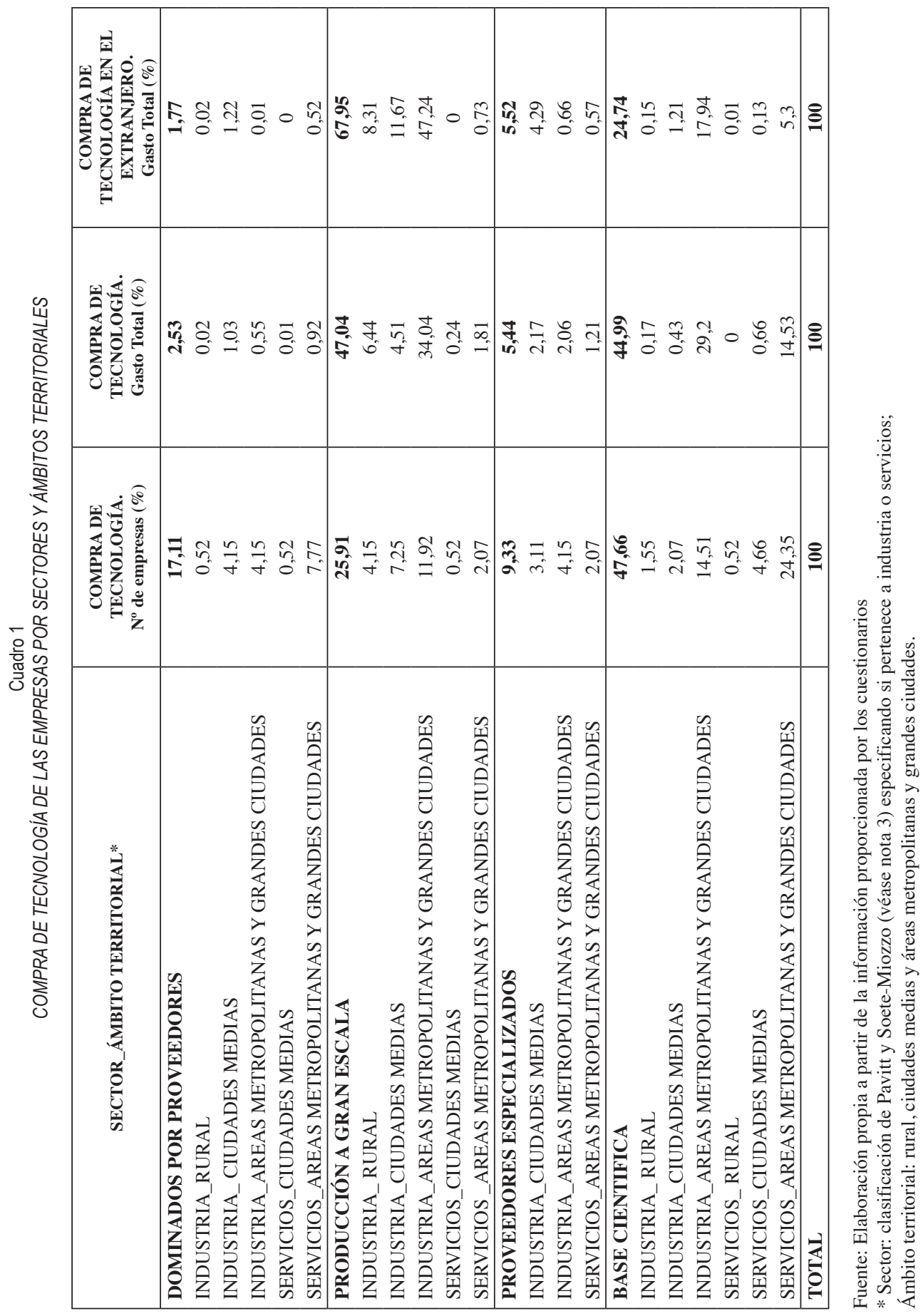


22,8\% de las firmas que han acometido alguna transacción (éstas efectúan el 9,4\% del gasto en tecnología por ser pymes), también los servicios avanzados (comienzan a desarrollarse) tienen una intensidad de I+D escasa. En el caso de las empresas ubicadas en áreas rurales $(6,74 \%)$, las cuales contabilizan el 10,6\% del gasto, buscan en dichos núcleos inputs de bajo coste (mano de obra, suelo) y suplen la falta de economías externas a través del tamaño empresarial y su interrelación con las empresas del grupo al que pertenecen.

La conjunción de estos hechos caracteriza unos sistemas locales, regionales y sectoriales de innovación de escaso grado de complejidad y de baja acumulación de conocimientos (Ruiz, 2005). En las relaciones de mercado de ámbito regional prevalece un tipo de intercambio de productos estandarizados, basados en tecnologías maduras y por la presencia de proveedores extranjeros que suministran dicha tecnología a un tejido productivo donde tienen un peso importante las empresas de producción a gran escala $(47,4 \%$ del gasto total en tecnología). Firmas que son las que mayor gasto han realizado en tecnología extranjera $(67,92 \%$ del total de ésta) (Cuadro 1).

\section{PRINCIPALES FACTORES DE LA COMPRA DE TECNOLOGÍA EN ANDALUCÍA}

De acuerdo con la metodología y el aparato teórico expuesto se aplicó la técnica de Análisis Factorial (AF) para determinar las interrelaciones entre variables utilizando el paquete estadístico de SPSS versión 15. Se obtuvieron 9 factores que explicaban el $65 \%$ de la varianza, resultando una comunalidad ${ }^{4}$ alta para todas las variables. Se empleó el método de extracción de factores de componentes principales ${ }^{5}$ y la rotación varimax con Kaiser, resultando un $\mathrm{KMO}^{6}$ de 0,735 , valor que permitía utilizar el AF con los datos muestrales (Cuadros 2 y 3 ).

El primer factor explica el 13,2\% de la varianza total y está asociado positivamente con las variables organización general, planificación estratégica, organización del proceso de trabajo (rutinas), desarrollo de procesos de creación, comunicación y registro de conocimientos, tecnología adquirida y propia. Según estas relaciones las empresas innovadoras dan mucha relevancia a la organización interna general y a la de los procesos de trabajo (revisión de rutinas, mecanismos de comunicación), valoran de forma importante el desarrollo de tecnología propia y la adquirida en el exterior y hacen planificación estratégica con registro de conocimientos.

El segundo factor concentra el 13,05\% de la varianza total. Se asocia positivamente con las variables «control de los equipos, tecnología adquirida, supervisión de la calidad, asistencia en diseño y desarrollo de procesos y formación de los empleados».Se interpreta por tanto que las empresas que adquieren tecnología llevan implícito en sus contratos de compra de

4 Comunalidad: proporción de la varianza explicada de una variable por el conjunto de los factores comunes. También se puede definir como proporción de varianza que puede ser explicada por el modelo factorial obtenido

5 Cuando se aplica este método la comunalidad de extracción inicial de cada variable es igual a 1 ya que se consideran todos los componentes o factores incluyendo la unicidad. No hace distinción entre varianza única y común. El primer factor explica la mayor parte de la varianza total; el segundo explicaría la mayor parte de la varianza restante

6 Kaiser-Mayer-Olkin (KMO) compara la magnitud de los coeficientes de correlación observados con la magnitud de los coeficientes de correlación parcial. Debe tomar un valor próximo a 1 . Los menores de 0,5 indican que no debe emplearse el AF con los datos muestrales empleados. 
tecnología acuerdos de control de los equipos, asistencia en diseño, etc.; es decir, adquieren también el know how además de la tecnología. El comprador no sólo necesita ésta última sino también debe procurarse las habilidades (know-how) para aplicar la tecnología en la producción y comercialización de los productos.

El tercer factor concentra el $6,8 \%$ de la varianza total y se relaciona positivamente con la variable «compra de tecnología en Europa» y negativamente con «localización de las empresas en ciudades medias y áreas rurales»; lo cual indica que las firmas innovadoras internacionalizadas que adquieren tecnología en Europa se ubican principalmente en las grandes ciudades y en áreas metropolitanas.

El cuarto factor representa el 5,9\% de la varianza total y está asociado negativamente con la variable «clasificación sectorial de Pavitt y Soete Miozzo» y positivamente con los recursos organizacionales. Cabría interpretar que las empresas que le dan mucho valor a los recursos de organización pertenecen a la rama industrial.

El quinto factor concentra el 5,9\% de la varianza total y se asocia positivamente con las variables «compra de tecnología llaves en mano e inversión en tecnología» y negativamente con «compra de tecnología de bienes de equipo». Es decir, las empresas que invierten en productos llaves en mano no adquieren bienes de equipo.

El sexto factor explica el 5,55\% de la varianza total y tiene que ver con las variables «porcentaje de facturación» y «capital europeo». Se interpreta que las empresas que tienen un alto porcentaje de facturación están participadas por capital europeo.

El séptimo factor acumula el 5,3\% de la varianza explicada y vincula las variables «mercado extranjero», «compra de tecnología en el mundo». Cabría entender que las empresas innovadoras internacionalizadas cuya venta se realiza mayoritariamente en el extranjero adquieren la tecnología también en el resto del mundo.

El octavo factor concentra el $4,8 \%$ de la varianza total, se asocia positivamente con las variables «importancia de las rutinas internas» y negativamente con «otras actividades innovadoras». Se interpreta que las empresas que le dan importancia a la organización de rutinas internas realizan actividades innovadoras a través de proyectos financiados.

El noveno factor explica el $4,7 \%$ de la varianza total y se relaciona positivamente con la variable «compra de patentes» y negativamente con «compra de otras tecnologías desincorporadas». Es decir, las empresas que adquieren patentes no compran otras tecnologías desincorporadas.

Cuadro 2

KMO Y PRUEBA DE BARTLETT

\begin{tabular}{|c|c|c|}
\hline \multicolumn{2}{|c|}{$\begin{array}{l}\text { Medida de adecuación muestral de } \\
\text { Kaiser-Meyer-Olkin. }\end{array}$} & ,735 \\
\hline $\begin{array}{l}\text { Prueba de esfericidad } \\
\text { de Bartlett }\end{array}$ & $\begin{array}{l}\text { Chi-cuadrado } \\
\text { aproximado } \\
\text { g }\end{array}$ & $\begin{array}{r}1250,286 \\
300\end{array}$ \\
\hline & Sig. & 000 \\
\hline
\end{tabular}

Fuente: Elaboración propia a partir de la información proporcionada por los cuestionarios 
Cuadro 3

MATRIZ DE COMPONENTES ROTADOS (A)

\begin{tabular}{|l|c|c|c|c|c|c|c|c|c|}
\hline & \multicolumn{7}{|c|}{ Componente } & $\mathbf{9}$ \\
\hline & $\mathbf{1}$ & $\mathbf{2}$ & $\mathbf{3}$ & $\mathbf{4}$ & $\mathbf{5}$ & $\mathbf{6}$ & $\mathbf{7}$ & $\mathbf{8}$ & $\mathbf{9}$ \\
\hline TIPO ORGANIZACIÓN & 0,784 & & & & & & & & \\
\hline ESTRATEGIA & 0,772 & & & & & & & & \\
\hline PROCESOS_CREACIÓN & 0,771 & & & & & & & & \\
\hline PROCESOS_RUTINAS & 0,765 & & & & & & & & \\
\hline TECNOLOGÍA_ADQUIRDA & 0,556 & & & & & & & & \\
\hline TECNOLOGÍA_PROPIA & 0,549 & & & & & & & & \\
\hline CALIDAD & & 0,872 & & & & & & & \\
\hline FORMACIÓN & & 0,864 & & & & & & & \\
\hline CONTROL DE EQUIPO & & 0,859 & & & & & & & \\
\hline ASISTENCIA DISEÑO & & 0,827 & & & & & & & \\
\hline COMPRA TECNOLOGÍA EN EUROPA & & & 0,767 & & & & & & \\
\hline LOCALIZACIÓN & & & $-0,727$ & & & & & & \\
\hline SECTOR PAVITTySOETTE & & & & $-0,681$ & & & & & \\
\hline RECURSOS ORGANIZACIONALES & & & & 0,663 & & & & & \\
\hline COMPRA DE PLANTAS LLAVE EN MANO & & & & & 0,742 & & & & \\
\hline COMPRA DE BIENES DE EQUIPO & & & & & $-0,634$ & & & & \\
\hline INVERSIÓN EN TECNOLOGÍA & & & & & 0,634 & & & & \\
\hline FACTURACIÓN & & & & & & 0,717 & & & \\
\hline CAPITAL PRIVADO UNIÓN EUROPEA & & & & & & 0,662 & & & \\
\hline MERCADO EXTRANJERO & & & & & & & 0,808 & & \\
\hline COMPRA TECNOLOGÍA EN RESTO DEL MUNDO & & & & & & & 0,649 & & \\
\hline OTRAS ACTIVIDADES INNOVADORAS & & & & & & & & $-0,809$ & \\
\hline RUTINAS INTERNAS EN LA EMPRESA & & & & & & & & 0,600 & \\
\hline COMPRA DE PATENTES & & & & & & & & & 0,740 \\
\hline OTROS TIPO DE COMPRA & & & & & & & & & $-0,629$ \\
\hline
\end{tabular}

Fuente: Elaboración propia a partir de los cuestionarios. Método de extracción: Análisis de componentes principales. (a) La rotación ha convergido en 13 iteraciones.

\section{COMPORTAMIENTO DE LAS EMPRESAS EN LA COMPRA DE TECNOLOGÍA Y SU INTEGRA- CIÓN EN EL TERRITORIO}

A través del análisis de conglomerados jerárquicos, y con las puntuaciones factoriales ${ }^{7}$ obtenidas mediante regresión (resultantes del análisis factorial) se identifican 6 grupos clúster. Después con el análisis de conglomerados de $\mathrm{K}$ medias ${ }^{8}$, se clasificó a cada empresa que

7 Son puntuaciones de los sujetos en cada uno de los factores resultantes de la extracción a fin de valorar la situación relativa de cada caso/sujeto/territorio

8 El análisis cluster de K-medias es una herramienta diseñada para asignar casos a un número fijo de grupos (clusters o conglomerados) cuyas características no se conocen aún pero que se basan en un conjunto de variables especificadas. Es muy útil cuando queremos clasificar un gran número de casos. El procedimiento del análisis cluster de K-medias empieza con la construcción unos centros de conglomerados iniciales. Podemos asignar éstos 
adquirió tecnología en función del número de grupos hallados, con el fin de obtener conglomerados de características similares.

Como las diferencias entre las 193 empresas de la muestra que realizaron compra de tecnología eran elevadas, a través del dendograma ${ }^{9}$ se llegó a una clasificación de 12 grupos con una distancia de unión de conglomerados de 5, destacando 4 pequeños grupos de empresas con una distancia de unión entre 10 y 25. Tras varias pruebas, se seleccionaron 6 grupos que ofrecían un nivel de diferenciación adecuado a través de una distancia de unión de conglomerados de 7,5. Por otra parte, el método de agrupamiento escogido fue el de vinculación inter-grupos, cuya diferencia entre dos conglomerados se calcula como la distancia promedio existente entre todos los pares de elementos de ambos grupos (Cuadro 4). A continuación, se analiza el comportamiento de cada uno de los grupos ante la compra de tecnología y su grado de integración en el tejido productivo. Cuestiones que se sintetizan de forma gráfica en la figura 1.

GRUPO 1. Constituye el 6,28\% de las empresas que han comprado tecnología $(8,69 \%$ del gasto total), están participadas por capital europeo $(72,16 \%)$, tienen una gran facturación (fact6) y un porcentaje de mercado extranjero relativamente alto $(19,38 \%$ de media de facturación). Son firmas de base científica, ubicadas principalmente en áreas metropolitanas (70\%); localizándose el resto (industriales) en ciudades medias, porque requieren economías externas. No basan sus capacidades en los recursos organizacionales (organización, proceso de trabajo o rutinas, procesos de creación, comunicación y registro de conocimientos, estrategia y relaciones formales e informales con otras empresas) (fact4).

Por el contrario, la fuente de sus capacidades está en los recursos humanos (cualificación del personal con un 4,58 de valoración sobre 5) y en la formación de éste $(3,75)$. No compran patentes (fact9) y la tecnología les viene transferida por la adquisición de bienes de equipo $(68,65 \%$ del gasto en tecnología) y por aquella otra que les cede de forma desincorporada la empresa matriz. Esta tecnología externa viene asociada a acuerdos de control de equipos, supervisión de la calidad y formación, de manera que al mismo tiempo que se adquiere el bien de equipo u otra tecnología la firma proveedora cede know-how $(21,42 \%)$ a la empresa compradora, tal como ocurre en países emergentes como por ejemplo Brasil (Oliveira, Jayme y Lemos, 2005.) Este flujo tiene origen en España (40,92\% del gasto total), Europa (38,24\%) y Norteamérica $(14,28 \%)$, y se compra a la industria de proveedores especializados $(38,29 \%)$ y a los servicios de base científica $(48,01 \%)$ para incorporar tecnologías punteras y modernizar sus procesos. Por ello conocen los cambios generados en el entorno extranjero a través del nuevo conocimiento adquirido, lo que les permite renovar continuamente su stock de conocimientos. Sin embargo, estas firmas no tienen relaciones con proveedores de tecnología

nosotros mismos o tener un procedimiento de selección de k observaciones bien situadas para los centros de conglomerados. Después de la obtención de los centros de los conglomerados: 1.- se asignan casos a los conglomerados basándose en la distancia de los centros de los conglomerados. 2.- Se actualizan las posiciones de los centros de los conglomerados basándose en los valores medios de los casos en cada conglomerado. Estos pasos se repiten hasta que cualquier reasignamiento de los casos haga que los conglomerados sean internamente más variables y externamente similares.

9 En este gráfico están representados los conglomerados y las etapas de fusión. Es de gran utilidad para evaluar la homogeneidad de los grupos y facilita la decisión sobre el número óptimo de conglomerados. 
ni de mercado en Andalucía, mostrando una escasa integración tecnológica y productiva en la región. Y por lo tanto sólo generan redes tecnológicas con el exterior.

El $70 \%$ de las firmas tienen departamento de I+D con un presupuesto medio de $468.244,4 €(1,27 \%$ de la facturación) obteniendo como resultado productos mejorados (76,6\%), y dedican a la adquisición de tecnología externa un 5,02\% de su facturación. En consecuencia, estas empresas otorgan una importancia media al desarrollo de tecnología propia y externa, y tardan más o menos un año en decidir adoptar esas tecnologías y 12 meses más en asimilarlas y desarrollar productos/procesos. Sin embargo, los competidores extranjeros son capaces de copiarles y adoptar la misma tecnología en un plazo de 7 meses. Las razones de este hecho obedecen a que los sistemas y componentes a elaborar precisan introducir en las firmas de base científica mayor diversidad de campos de conocimiento debiendo cooperar más para ello o adquirirlos en el exterior. Conocimiento que deben asimilar y aplicar en el transcurso de un periodo de tiempo más corto del que ellos habitualmente utilizarían. Además, se precisa tener mayor destreza de ingeniería de producto y de diseño, tal como sucede en diversos países sudamericanos (Arvanitis, 2003). De ahí que estas necesidades de aprendizaje rápido y su falta de capacidad de absorción es lo que explica la diferencia entre los competidores y las empresas andaluzas.

GRUPO 3. Supone el $30,8 \%$ de las empresas y el 11,49\% del gasto de compra tecnológica. Se trata fundamentalmente de firmas localizadas en áreas metropolitanas $(74,19 \%$ por la necesidad de economías externas), son de capital regional $(83,49 \%)$ y nacional $(11,54 \%)$, en las que predominan las de servicios avanzados de base científica. Este mismo comportamiento muestra las empresas ubicadas en ciudades medias $(19,35 \%)$, bien sean de servicios o industriales. El conglomerado se diferencia de los demás por la compra de tres tipos de tecnología: bienes de equipo, plantas llaves en mano y software (fact5 y 9), con un esfuerzo en inversión tecnológica de un 4,27\% de su facturación. Sin embargo, esta compra de tecnología no está asociada a contratos de supervisión de calidad, de control de equipos, de diseño y de formación (fact2), otorgándose una valoración inferior a 1 en una escala de 1 a 5 . Pues, las capacidades de estas empresas se basan en la importancia que se le da a los recursos humanos $(55,57 \%$ frente los organizacionales y tecnológicos 44,4\%), principalmente a la cualificación $(3,64$ sobre 5) y a la formación del personal $(3,32)$. Estos hechos suceden también en las empresas del País Vasco (García, Puch, García y Gutiérrez, 2011).

Los flujos de compra de tecnología se desarrollan sobre todo en el interior de la región (48,12\% del gasto), en España $(25,60 \%)$, y en menor medida con el extranjero (Europa $(9,21 \%)$ y Norteamérica $(17,05 \%)$, cobrando fuerza el factor de proximidad física, cultural y social. Y un $22,5 \%$ de estas firmas complementan la adquisición de tecnología externa realizando acuerdos con socios tecnológicos españoles y norteamericanos. Estas empresas tardan aproximadamente unos 5 meses en decidir adoptar las tecnologías externas y 15 meses más en asimilarlas y desarrollar productos/procesos derivadas de éstas; mientras que los competidores extranjeros y nacionales son capaces de copiarles y adoptar la misma tecnología en 8,64 meses debido a ser productos/proyectos personalizados, cuya metodología lleva tiempo en desarrollarse, pero es fácil de copiar porque estos proyectos/ informes en la mayoría de los casos son públicos.

Así pues, las empresas de este grupo adquieren tecnología en el ámbito regional, detectándose en Andalucía la existencia de un conjunto de empresas proveedoras de servicios 
avanzados, de bienes de equipo industriales especializados (19,69\% sobre total gastos compra tecnología del grupo), de software $(40,95 \%)$ y de marcas comerciales $(11,58 \%)$. Por lo que existen flujos de transferencia de conocimiento al tejido productivo andaluz, vía proveedores y clientes, generando una fuerte integración territorial. Por otra parte, realizan actividades de I+D (el 50,84\% de ellas tiene departamento de I+D y gastan anualmente $456.228 €$ ) e ingeniería o diseño, con un presupuesto medio de innovación (no asociado a proyectos) muy elevado (2.757.092€). De ahí que este conjunto de empresas andaluzas hayan logrado de una parte, diferenciar sus productos $(64,40 \%)$ con innovaciones de mejora $(66,37 \%)$ que les permite ser competitivos en el mercado europeo (15,52\% de la facturación) y en el resto del mundo $(7,34 \%)$. Y de otra, desarrollar una importante tecnología propia (patentes, modelos de utilidad, software, know-how, marcas, etc.) interrelacionada con una cualificación elevada de los recursos humanos. El comportamiento innovador de este grupo es semejante al de las empresas servicios avanzados de Méjico Distrito Federal (Arroyo y Cárcamo, 2009).

GRUPO 4. Significa el 36,12\% de las firmas y el 17,33\% del gasto de compra tecnológica. Es un grupo formado por empresas de capital regional (85,08\% de su capital), y algunas participadas de capital del resto de España (12,13\%); y son principalmente industriales $(72,44 \%)$, donde destacan las de producción a gran escala $(27,54 \%)$ y las de base científica $(21,74 \%)$. Tienen una fuerte presencia en ciudades medias $(30,43 \%)$ y zonas rurales $(11,59 \%)$ aunque el $57,97 \%$ se ubican en áreas metropolitanas.

La tecnología adquirida es sobre todo de bienes de equipo y software como muestran los fact5 y 9, que supone por término medio el $89,44 \%$ de sus gastos en tecnología. Se compra en Europa (31\% del total de gastos) (fact3), España $(31,75 \%)$ y Andalucía $(23,57 \%)$. Y se realiza la adquisición principalmente a sectores industriales, particularmente al de proveedores especializados, no estableciéndose acuerdos para la implantación de la tecnología (fact2). Estas empresas tardan aproximadamente 9 meses en decidir adoptar esas tecnologías externas y 19 meses más en asimilarlas y desarrollar productos/procesos derivadas de éstas. Sin embargo, en menos de 6 meses los competidores extranjeros y nacionales son capaces de copiarles y adoptar la misma tecnología debido a que han desarrollado una mayor base tecnológica que las firmas andaluzas. En el caso de los sectores de base científica, Andalucía, España y otros países europeos (Bélgica, Italia, Holanda, Suecia, etc.) están perdiendo posiciones en el mercado mundial frente a naciones como China, India, Corea del sur, Hungría y Méjico (Jordá, 2009). Así pues, se necesita reforzar las inversiones de I+D y las de tecnología externa desincorporada para recuperar los puestos perdidos y reducir la diferencia en cuanto a capacidad de absorción entre los competidores y la firma andaluza.

Un 60\% de las empresas tienen departamento de I+D (presupuesto medio: $405.287 €$ ) y el resto de diseño e ingeniería para desarrollar productos diferenciados $(65,94 \%)$ y mejorados $(68,84 \%)$. El $27 \%$ de ellas disponen de socio tecnológico para aportarles I+D $(2,30 \%$ de la facturación), y la capacidad organizacional de la empresa juega un papel relevante (fact4 y 1). Además, la cualificación (4,2 sobre 5) y la formación del personal (3,71 sobre 5) toman valores altos, la organización de los procesos de trabajo, medidos a través de las rutinas, alcanza un índice de 4,36 (entre 1 y 5). De la misma manera que la tecnología adquirida consigue un valor del 4,14 sobre 5, asociado a la compra de bienes de equipo para modernizar los procesos productivos. Luego, estas empresas, en el contexto andaluz, tienen experiencia en la organización de los procesos que van asociados a la I+D (organización del 
trabajo, organización general de la empresa, política formativa y estrategia), pero éste en parte lo adquieren a los socios tecnológicos. Este comportamiento innovador también se ha confirmado en las firmas manufactureras del área metropolitana de Méjico Distrito Federal (Padilla y Martín, 2003).

Todo ello les permite ser competitivos y abarcar a partes iguales un mercado regional y nacional (35,5 y 34,71\%). Este grupo muestra sinergias vía clientes en Andalucía; sin embargo, a través de proveedores estas relaciones son muy escasas ya que sólo realizan un $7,6 \%$ de gastos con proveedores de mercado regionales.

GRUPO 5. Agrupa el 25,65\% de las empresas que han adquirido tecnología y representa el 52,27\% del gasto de compra tecnológica; la mayoría son de capital regional (participación media $61,5 \%)$, nacional $(16 \%$,), europeo $(6,38 \%)$ y del resto del mundo $(8,06 \%)$. Fundamentalmente se sitúan en áreas metropolitanas $(81,63 \%)$ y en menor medida en ciudades medias $(12,75 \%)$ y zonas rurales $(8,16 \%)$. Y son firmas de base científica (industria y servicios) e industriales de producción a gran escala.

A través de la compra de tecnología han firmado acuerdos de supervisión de la calidad $(3,43$ de media sobre 5$)$, formación $(3,31)$, control de equipos $(3,35)$, asistencia en diseño $(3,15)$ (fact2) para implantar en su proceso productivo nuevas tecnologías y/o desarrollar nuevos productos: software, asistencias técnicas, patentes, know-how y bienes de equipo. Tecnologías que se han comprado en España $(38,08 \%$ de los gastos totales), Andalucía $(26,8 \%)$, Europa $(24,35 \%)$ y Norteamérica $(9,49 \%)$. Estas empresas tardan unos 14 meses en tomar la decisión sobre que tecnología externa adoptar, en aplicarla 8,14 meses, en generar el producto 6 y unos 7 en ser copiados por los competidores nacionales y extranjeros. En el caso de los sectores de producción a gran escala andaluces sucede que las empresas están sujetas a cambios tecnológicos cada vez más rápidos precisando una innovación continua, además de necesitar implementar conocimientos afines en diversos campos. Sin embargo, muchas de estas firmas no son capaces de desarrollar habilidades de aprendizaje tecnológico rápido para seguir el ritmo tecnológico mundial. Marta Novic (2002) comprueba que en diversos sectores manufactureros de Argentina ocurre algo semejante.

La compra de tecnología del grupo se diferencia del resto por la adquisición de bienes de equipo (54,54\% de gasto) y por el elevado peso relativo de las patentes adquiridas $(12,75 \%)$. Un $20 \%$ de las empresas complementan la adquisición de tecnología realizando acuerdos con socios tecnológicos europeos y norteamericanos (6,4\% de la facturación) a fin de mejorar su base tecnológica. Este comportamiento viene asociado a un importante esfuerzo innovador ya que dedican a I+D y a otras actividades innovadoras un presupuesto medio anual alto (1.961.658 € que significa el 51,32\% de los gastos de innovación), al igual que sucede en diversos países de la Unión Europea (Rodríguez, 2007). Por otra parte, las firmas de este grupo valoran por encima de la media las tecnologías adquiridas (3, 59 sobre 5), la formación del personal $(3,88)$, la cualificación de éste $(4,33)$ y la organización $(3,51)$. De ahí que los resultados tecnológicos arrojen: 1) una gran diversidad y un elevado número de tecnologías desincorporadas registradas (patentes, modelos de utilidad, plantas llaves en mano y software); y 2) generan productos diferenciados (65,64\% de media de su facturación), de los que un $43 \%$ son nuevos, y así poder ser competitivos, en base a la calidad de numerosas marcas, en el mercado nacional $(38,64 \%)$, europeo $(13,64 \%)$ y en el resto del mundo $(10,7 \%)$. 
Estas firmas compran los bienes de equipo y las patentes a la industria de producción a gran escala $(22,13 \%)$, a la de proveedores especializados $(20,83 \%)$ y a los servicios avanzados (36,82); generan sinergias tecnológicas en la región (26,6\% del gasto en tecnología), y a través de los proveedores (10,7\% del total que se realiza con empresas andaluzas) con empresas de producción a gran escala $(8,78 \%)$ y de base científica $(1,83 \%)$, luego hay integración de estas firmas en la CCAA.

GRUPOS 2 Y 6. Por otra parte, los grupos 2 y 6 cuentan con una sola empresa cada uno, si bien, dado que destacan de forma significativa en algunos factores hemos optado por estudiarlas aparte, después de haber confirmado las grandes diferencias existentes con el resto de grupos y entre sí:

1. Empresa perteneciente al clúster 2. Ubicada en una ciudad media, es multinacional de capital europeo y del resto del mundo, con un mercado mundial, y se caracteriza por la compra de tecnología en el extranjero, en particular, en el resto del mundo (fact7) y Europa (fact3). Tiene recursos humanos, organizacionales y tecnológicos eficientes y avanzados (fact1 y fact4) que «no les obliga a firmar» acuerdos de control y supervisión de calidad de los equipos adquiridos, etc., para implantar dicha tecnología. Se trata, pues, de una empresa perteneciente a un sector donde las marcas comerciales son importantes $(83,58 \%$ del gasto en tecnología) y los bienes de equipo $(16,41 \%)$. La inversión en tecnología es alta ( $8 \%$ de su facturación), pero no realizan gastos en $\mathrm{I}+\mathrm{D}$, sino que efectúan otras actividades innovadoras fuera de proyectos, lo que sólo les lleva a mejorar su productos (75\% de su facturación).

2. Empresa del grupo 6.- Localizada en un área metropolitana, se caracteriza desde el punto de vista tecnológico por el desarrollo de su propia tecnología, aprovechando todas las capacidades y recursos que le otorga la pertenencia a un grupo empresarial internacional del sector de producción a gran escala con sede en Europa. El grupo le proporciona numerosas patentes y marcas, y la I+D necesaria para desarrollar sus productos, realizando la propia empresa otras actividades de innovación más ligadas al proceso de producción (fact7). A ello se une, pero con mucha menor importancia y cuando es necesaria, la compra de tecnología externa, tomando la decisión de adoptarla de forma rápida (unos 3 meses), así como en apli-

Cuadro 4

CENTRO DE LOS CONGLOMERADOS FINALES

\begin{tabular}{|c|c|c|c|c|c|c|}
\hline & \multicolumn{6}{|c|}{ Cluster } \\
\hline & 1 & 2 & 3 & 4 & 5 & 6 \\
\hline REGR factor score 1 for analysis 1 & 0,18190 & 1,41058 & $-0,33634$ & 0,17797 & 0,10013 & $-0,93575$ \\
\hline REGR factor score 2 for analysis 1 & 0,25319 & $-2,05515$ & $-0,57833$ & $-0,39100$ & 1,19873 & 1,37941 \\
\hline REGR factor score 3 for analysis 1 & 0,29564 & 3,42706 & $-0,42062$ & 0,36966 & $-0,19815$ & 2,04460 \\
\hline REGR factor score 4 for analysis 1 & $-0,14385$ & $-3,45951$ & $-0,39437$ & 0,49395 & $-0,13717$ & 1,09230 \\
\hline REGR factor score 5 for analysis 1 & 0,13661 & $-1,62632$ & 0,57616 & $-0,61296$ & 0,05601 & 5,54314 \\
\hline REGR factor score 6 for analysis 1 & 2,92894 & 1,41010 & $-0,23062$ & $-0,18133$ & $-0,17872$ & $-1,68204$ \\
\hline REGR factor score 7 for analysis 1 & 0,08410 & 8,92825 & $-0,03244$ & $-0,18294$ & 0,06035 & 1,64227 \\
\hline REGR factor score 8 for analysis 1 & 0,33478 & $-1,09442$ & $-0,22521$ & 0,36119 & $-0,35345$ & 2,76177 \\
\hline REGR factor score 9 for analysis 1 & $-0,07230$ & 0,85991 & $-0,44560$ & 0,06378 & 0,43483 & 0,59061 \\
\hline
\end{tabular}

Fuente: Elaboración propia a partir de la información proporcionada por los cuestionarios. 


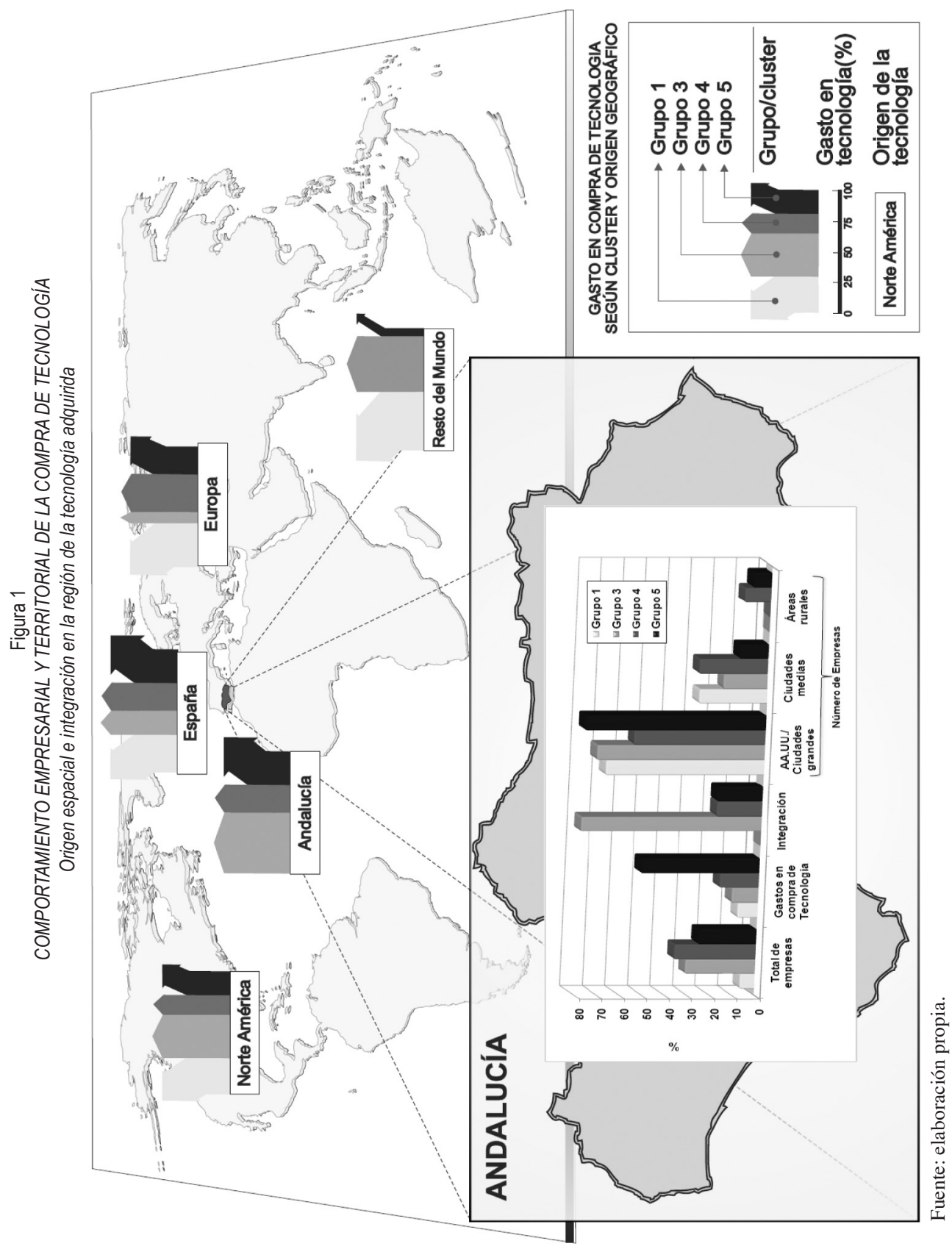


carla y desarrollar el producto (6 meses). En los últimos tres años se ha acometido un gran esfuerzo tecnológico (10\% de su facturación) para comprar una planta llave en mano (fact5), software y bienes de equipo. Estas transacciones se han realizado con proveedores europeos (91\% del gasto realizado en tecnología externa) y norteamericanos (fact3 y 7), con los que han establecido acuerdos para la transmisión y absorción del know-how ligada a dicha tecnología (fact2). Todo ello, junto con la alta cualificación del personal y una organización del trabajo con una elevada rotación (fact8). Factores que le facilitan competir en el mercado nacional $(63,5 \%)$ y europeo $(36,5 \%$ de su facturación) en productos nuevos y mejorados (fact6). Igualmente, esta estrategia innovadora es aplicada por numerosas empresas de países europeos como por ejemplo Holanda (Los y Timmer, 2005).

\section{CONCLUSIONES}

En Andalucía, el número de empresas innovadoras es reducido. En éstas, y sobre todo en las internacionalizadas, juega un importante papel las actividades internas de innovación (I+D y otras actividades innovadoras) y las externas (compra de tecnología e I+D). Sin embargo, en términos de resultados, las innovaciones introducidas en los procesos y en los productos no han atenuado las diferencias tecnológicas existentes con los competidores extranjeros porque gran parte de la tecnología adquirida es para modernizar el proceso productivo y el presupuesto de I+D es bajo comparado con la media nacional (791.754,5€) salvo en el cluster 5. Esto obedece a que el tejido empresarial innovador está formado sobre todo por empresas: a) de producción a gran escala donde la innovación deriva de las necesidades de aminorar costes, elaborándose productos destinados a grandes mercados con cierto grado de estandarización; y b) de base científica que comprende firmas con alta capacidad tecnológica y otras de bajo valor añadido.

Para muchas empresas de estos sectores es más rentable imitar de forma creativa las innovaciones desarrolladas por otras, pues, la fuerte competencia y el bajo nivel de acumulación de conocimientos de éstas hacen inviable trasladar a los precios de los productos el coste de la innovación. Estas suelen ser de proceso (bienes de equipo) lo que explica que emigren con facilidad a los competidores. De ahí que para elevar el nivel tecnológico de la región haya que incrementar el gasto en innovación de producto, la base de conocimiento empresarial y diversificar el tejido con empresas de media-alta y alta tecnología (OCDE, 1997).

El número de empresas que han adquirido tecnología en el extranjero (incluidas las internacionalizadas) es reducido. Su capacidad de compra viene definida, según los resultados del análisis factorial, por cuatro grupos de componentes:

1. La existencia de capacidades o recursos, humanos, organizacionales y tecnológicos correlacionados con la generación de I+D (factores 1,4 y 8). En la actualidad, la I+D sistemática y a través de proyectos exige introducir un conjunto de procesos interrelacionados: cambios en la organización general de la empresa y en el trabajo, e incorporar una política formativa e incentivadora. El tipo de transacción tecnológica puede ser directa a través de la compra de bienes de equipo, plantas llave en mano, patentes, know how, etc.; o de forma indirecta por acuerdos de mantenimiento de equipos y otras tecnologías (factores 2, 5 y 9). 
2. Las variables territoriales (localización y alcance territorial del mercado - exportación-) están en relación con la magnitud de la compra de tecnología en el extranjero (Europa o resto del mundo) (factores 3 y 7).

3. Las características estructurales de las empresas (origen del capital y tamaño según facturación) influyen en el volumen del gasto de compra tecnológica (fact3).

Estos factores, y a partir del análisis cluster, diferencian la existencia de un grupo muy numeroso (cluster 3), donde predomina la pequeña empresa de servicios de base científica, con poco gasto en tecnología extranjera pues el conocimiento reside en la alta cualificación de sus recursos humanos. Estas empresas son claves (inputs intermedios) para la transferencia tecnológica entre firmas locales, sobre todo en las áreas metropolitanas donde se localizan, ya que establecen intensas relaciones con clientes, pero no para incrementar el nivel tecnológico a una cota semejante al de los países desarrollados.

Los clusters 4 y 5 (dos tercios de las empresas encuestadas y casi el $70 \%$ del gasto en tecnología externa), indican como la compra de ésta última contribuye al desarrollo del territorio. En Andalucía, el proceso de innovación empresarial, la transferencia tecnológica y el éxito en el mercado está asociado a la concentración geográfica de empresas, por lo que los ámbitos territoriales que se benefician de dichos procesos son las áreas metropolitanas en correlación con las economías externas. Es el cluster 5, el que más ha invertido en I+D y en tecnología interna y externa (extranjero), el que más valor otorga a la cualificación y en definitiva, a los conocimientos explícitos y tácitos para alcanzar importantes resultados tecnológicos y ser competitiva en el mercado. No obstante, sería necesario: a) disminuir los tiempos de absorción de la tecnología a nivel empresarial para crear más ventaja competitiva frente a competidores en sectores de tecnología madura como son las de producción a gran escala; y b) reducir la elevada concentración territorial de estas empresas, y por lo tanto, favorecer el desarrollo de economías externas en ámbitos de ciudades medias que permita atenuar las diferencias territoriales existentes en la región en términos de innovación y desarrollo.

Desde la óptica de las sinergias que generan las empresas compradoras de tecnología, mediante redes territoriales y sectoriales con proveedores, destacan los clusters 4 y 5 que son los que más potencialidad manifiestan para transferir tecnología al territorio. Por un lado, la compra de tecnología en Europa y en el resto del mundo (70\% de las empresas) está ligada a variables espaciales (áreas metropolitanas y alcance del mercado de exportación) en los grupos 1,4 y 5 y en las dos empresas que muestran comportamientos diferentes al resto. Así, las economías externas que se dan en las áreas metropolitanas (comunicaciones, cualificación de la mano de obra, concentración y competencia entre empresas, etc.) favorecen la interacción de información y conocimiento de éstas a escala mundial. La empresa innovadora metropolitana suele recurrir a proveedores internacionales, líderes tecnológicos en sus respectivas ramas, para incorporar aquellas tecnologías punteras que modernicen sus procesos productivos y aumenten el grado de innovación en términos de productos y procesos.

Pero también las actividades de innovación, especialmente las ligadas a la transferencia de conocimiento tácito, necesitan ser realizadas cara a cara, cobrando fuerza el factor de proximidad geográfica, cultural y social. Así, después de analizar los vínculos con proveedores locales mediante transacciones tecnológicas y de mercado, se demuestra que una parte de las empresas que han adquirido tecnología en Andalucía y España la transfieren al terri- 
torio. El análisis cluster identifica dos grupos de empresas $(61,77 \%$ del total) que asocian la adquisición externa con: 1) el desarrollo de tecnología propia; y 2) la capacidad de transferir tecnología comprada y/o generada por la empresa a sus proveedores (grupos 4 y 5). Los intercambios tecnológicos y/o de mercado de las empresas que han comprado tecnología con proveedores andaluces suponen el 19\% del total (medidos en valores monetarios). Cabe destacar que:

a. Las empresas del grupo 4 representan el 7,6\% de estos intercambios y transfieren principalmente tecnología de bienes de equipo, intangibles e inputs electrónicos; y

b. Las firmas del grupo 5 suman el 10,7\% de las transacciones, estableciendo flujos fundamentalmente con la industria de producción a gran escala y de base científica para intercambiar inputs electrónicos e intangibles.

Así pues, los datos indican que las firmas compradoras de tecnología adquieren un volumen reducido de insumos locales o regionales ya que Andalucía posee un escaso número de proveedores locales de tecnología. Esto se debe a que: a) las firmas innovadoras, y en particular las que poseen capacidad de adquisición tecnológica, tienen posibilidad de abastecerse de inputs estandarizados internacionales en el propio territorio debido a la localización de empresas proveedoras extranjeras; y b) Andalucía se caracteriza por un sistema regional de innovación de escaso grado de complejidad tecnológica incapaz de suministrar tecnología a las empresas compradoras de ésta.

\section{BIBLIOGRAFÍA}

ARROYO, P. y CÁRCAMO, L. (2009): «El desarrollo de KIBS en Méjico. El sector servicios en el contexto de la economía del conocimiento». Economía y sociedad, vol. XIV, $\mathrm{n}^{\mathrm{o}} 23$, pp. 65-78.

ARVANITIS, R. (2003): «Science and technology policy» en Knowledge for Sustainable Development-An Insight into the Encyclopedia of Life Support Systems (Volume 3). UNESCO (Ed.). Paris, France, UNESCO Publishing/Eolss Publishers, pp. 811-848.

CANO, G. (2002): Las comarcas andaluzas. Sevilla, Ediciones Tartessos.

CARAVACA, I et al. (2005): «Hacia una tipología de ciudades según su capacidad innovadora. Aplicación a Andalucía» en A Geografía Ibérica no Contexto Europeu: Libro de Resumo Do X Coloquio Ibérico de Geografía. Coloquio Ibérico de Geografía. Num. 10. Évora (Portugal). Universidade Do Evora, pp. 119-120.

COHEN, W.M.; LEVINTHAL, D.A. (1990): «Absorptive-capacity- A New Perspective on Learning and Innovation». Administrative Sience Quarterl, Vol. 35, n 1, pp. 128-152.

DEMATTEIS, G. (1995): «Global networks, local cities». Flux, nº 15, pp. 17-33.

DIXÓN, N. (2000): Common knowledge: how companies thrive by sharing what they know. Boston, Harvard Business School, 336 pp.

FAGEBERG, J. (1994): «Technology and International Differences in Growth Rates». Journal of Economic Literature, vol. XXXII, n 3, pp. 17-26.

FERNÁNDEZ, E. (2005): Estrategia de Innovación, Madrid, Thomson.

FREEMAN, C.H. (994): «Innovation and Growth» en DOGSON/ ROTHWELL(1994): Innovation and Size of Firm. 
GANAU, J. Y VILAGRASA, J. (2003): «Ciudades medias en España. Posición en la red urbana y procesos urbanos recientes». Ciudades, arquitectura y espacio urbano. Barcelona, Caja Rural Intermediterránea, pp. 37-73.

GARCÍA, R.; PUCH, L.; GARCÍA, E.; GUTIÉRREZ, F. (2011): «Inversión e innovación en el País Vasco». Papeles de Economía Española, n ${ }^{\circ}$ 127, pp. 145-156.

HATZICHRONOGLOU, T. (1997): «Révision des classifications des secteurs et des produits de haute technologie «, Éditions OCDE , http://dx.doi.org/10.1787/050148678127

INE (2002): «Sectores de alta, media y baja tecnología» en http//www. ine.es

JIMÉNEZ, M.; MOLINA, L.; GARCÍA, V. (2009): «Capacidad de absorción del conocimiento: medición». Feg Working Papers Series, n 2/09, Facultad de Ciencias Económicas Universidad Granada.

JORDÁ, R. (2009): «Empresas y sectores innovadores» en Vallés, J. (Dir.): Economía Española, Madrid, Mc Graw Hill.

JORDÁ, R; RELAÑO, R. (2009): «Binomio innovación-internacionalización y su relación con la estrategia en las empresas innovadoras localizadas en Andalucía», en Scripta Nova. Vol. XIII, núm. 300. Disponible en http: // www.ub.edu/geocrit/nova.htm

JORDÁ, R.; RUIZ, F. (2009): «Comportamiento innovador de las empresas internacionalizadas por ámbitos territoriales en Andalucía. Flujos y Sistemas». Boletín de la Asociación de Geógrafos Españoles, n ${ }^{\circ}$.50, pp. 315-348.

KIM. L. (2000): Technology, Learning and Innovation [Tecnología, aprendizaje e innovación] (en colaboración con Richard Nelson). Cambridge University Press.

LANKHUIZEN, M. (2006): «Shifts in foreign trade, competitiveness and growth potential: from Baltics to Bal-techs». Rev Econ-papers, vol. 35, pp. 9-29.

LICHTENBERG, F.; SIEGEL, D. (1991): «The Impact of R\&D Investment on ProductivityNew Evidence Using Linked R\&D-Lrd Data». Economy Inquery, Vol. XXIX (April), pp. 38-49.

LÓPEZ, J. (2008): «La Industria Aeronáutica de Andalucía. Transferencia de Conocimientos e Innovación en las Empresas». Cuadernos del IDR. Núm. 39, 102 pp.

LOS, B. y TIMMER, M. (2005): «The appropiate technology explanation of productivity groth differentials: an empirical approach» Journal of development Economics, vol. 77, $\mathrm{n}^{\mathrm{o}} 2$, pp. 19-27.

LUCENDO, A.L. (2009): «Recursos tecnológicos y capacidades innovadoras en las empresas industriales andaluzas». Cuadernos de Geografía, $\mathrm{n}^{\circ}$ 85, pp. 55-82.

MAILLAT, D. Y KEBIR, L. (1998): «Learning region et systèmes territoriaux de production». Working Paper IRER 9802a, Université de Neuchâtel, Neuchâtel.

MÉNDEZ, R. (Dir.) (2007): Dinámica de las ciudades medias de Andalucía. Sevilla, Instituto de Estadística de Andalucía. 134 pp.

NOVIC, M. (2002): «Aprendizaje y conocimiento como ejes de competitividad. Capacitación e innovación en dos tramas productivas de la industria manufacturera argentina» en http://www.redmercosur.org

OLIVEIRA, F.; JAYME, F.; LEMOS, M. (2006): «Increasing returns to scale international diffusion». World Development, vol. 34, nº 1, pp. 75-88.

PADILLA, S. y MARTÍN, L. (2003): «Tremec-Chysler: una experiencia exitosa de innovación en Méjico». Revista Espacios, vol. 24, no 3 pp. 1-20. 
PAVITT, K. (1984): «Sectoral Patterns of technical change: towards a taxonomy and a theory». Research Policy, n 13, pp. 343-373.

PLA, J. Y LEÓN, F. (2004): Dirección de empresas internacionales. Pearson-Prentice Hall, Madrid.

RODRIGUEZ, D. (2007):«Concentración espacial y sectorial de la industria en la U.E.». Papeles de Economía Española, n ${ }^{\circ} 112$, pp. 27-38.

ROOT, F.R. (1994): Entry Strategies for Internacional Markets. Nueva York .Lexington Books.

RUIZ, F. (2005): I+D y Territorio. Análisis y Diagnóstico de la innovación empresarial en Andalucía. CES-A. Junta de Andalucía, Sevilla.

SOETE, L. Y MIOZZO, M. (1989): «Trade and development in services. A technological perspective». Working Paper, nº. 89-031, Merit, Maastricht.

ZAHRA, S. Y GEORGE, G. (2002): «Absorptive capacity: A review, reconceptualization and extension». Academy of Management Review, vol. 27, nº 2, pp. 185-203. 